Factors predicting chronic pain after open inguinal hernia repair : a regression analysis of randomized trial comparing three different meshes with three fixation methods (FinnMesh Study)

Matikainen, $\mathrm{M}$.

2018-10

Matikainen , M , Aro , E , Vironen , J , Kössi , J , Hulmi , T , Silvasti , S , Ilves , I , Hertsi , M , Mustonen , K \& Paajanen , H 2018, ' Factors predicting chronic pain after open inguinal hernia repair : a regression analysis of randomized trial comparing three different meshes with three fixation methods (FinnMesh Study) ' , Hernia , vol. 22 , no. 5 , pp. 813-818 . https://doi.org/10.1007/s1002

http://hdl.handle.net/10138/251526

https://doi.org/10.1007/s10029-018-1772-6

publishedVersion

Downloaded from Helda, University of Helsinki institutional repository.

This is an electronic reprint of the original article.

This reprint may differ from the original in pagination and typographic detail.

Please cite the original version. 


\title{
Correction to: Factors predicting chronic pain after open inguinal hernia repair: a regression analysis of randomized trial comparing three different meshes with three fixation methods (FinnMesh Study)
}

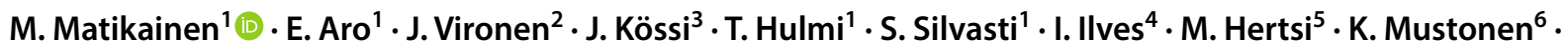 \\ H. Paajanen ${ }^{6}$
}

Published online: 6 June 2018

(c) Springer-Verlag France SAS, part of Springer Nature 2018

\section{Correction to: Hernia}

https://doi.org/10.1007/s10029-018-1772-6

In the original publication, co-authors affiliations were incorrect. The correct information is given below.

M. Matikainen, Department of Surgery, North Karelia Central Hospital, Tikkamäentie 16, 80210 Joensuu, Finland

E. Aro, Department of Surgery, North Karelia Central Hospital, Tikkamäentie 16, 80210 Joensuu, Finland

J. Vironen, Department of Surgery, Helsinki University Hospital, Turuntie 150, 02740 Espoo, Finland

J. Kössi, Department of Surgery, Paijat-Hame Central Hospital, Keskussairaalankatu 7, 15850 Lahti, Finland

T. Hulmi, Department of Surgery, North Karelia Central Hospital, Tikkamäentie 16, 80210 Joensuu, Finland

S. Silvasti, Department of Surgery, North Karelia Central Hospital, Tikkamäentie 16, 80210 Joensuu, Finland

The original article can be found online at https://doi.org/10.1007/ s10029-018-1772-6.

M. Matikainen

markku.matikainen@siunsote.fi

1 Department of Surgery, North Karelia Central Hospital, Tikkamäentie 16, 80210 Joensuu, Finland

2 Department of Surgery, Helsinki University Hospital, Turuntie 150, 02740 Espoo, Finland

3 Department of Surgery, Paijat-Hame Central Hospital, Keskussairaalankatu 7, 15850 Lahti, Finland

4 Department of Surgery, Mikkeli Central Hospital, Porrassalmenkatu 35, 50100 Mikkeli, Finland

5 Department of Surgery, Savonlinna Central Hospital, Keskussairaalantie 6, 57120 Savonlinna, Finland

6 Department of Surgery, Kuopio University Hospital, Puijonlaaksontie 2, 70210 Kuopio, Finland
I. Ilves, Department of Surgery, Mikkeli Central Hospital, Porrassalmenkatu 35, 50100 Mikkeli, Finland

M. Hertsi, Department of Surgery, Savonlinna Central Hospital, Keskussairaalantie 6, 57120 Savonlinna, Finland

K. Mustonen, Department of Surgery, Kuopio University Hospital, Puijonlaaksontie 2, 70210 Kuopio, Finland

H. Paajanen, Department of Surgery, Kuopio University Hospital, Puijonlaaksontie 2, 70210 Kuopio, Finland 